\title{
Cognitive masking: The disruptive effect of an emotional stimulus upon the perception of contiguous neutral items*
}

\author{
MATTHEW HUGH ERDELYI \\ and \\ ANAT GORDON APPELBAUM \\ Douglass College, Rutgers University \\ New Brunswick, N.J. 08903
}

The detection of a very briefly presented (200-msec) configuration of eight neutral stimuli was inferior when the ninth member of the stimulus array was an emotional rather than a neutral item. This outcome suggests that the symbolic meaning of portions of a stimulus may disrupt the processing of adjacent stimulus components in a single-fixation exposure of the whole stimulus

Behavioral as well as physiological evidence accumulating over the last few decades converges on the conclusion that perception is a highly interactive set of processes. It is now well known, for example, that the activation of a single visual receptor may have inhibiting as well as enhancing effects upon the later-stage processing of another receptor's signals (e.g., Cornsweet, 1970; Lindsay \& Norman, 1972; Ratliff, 1965; von Békésy, 1967). Complex neurological programs built upon such simple enhancement-inhibition interactions are now assumed to underlie the selective systems, at various levels of visual processing, capable of extracting specific types of stimulus features, such as slits, holes, edges, specific movements, specific slants, etc. Such interactions, moreover, are not restricted to topographic relationships. The considerable research on backward and forward masking (metacontrast, paracontrast, etc.) (e.g., Kahneman, 1968; Raab, 1963; Weisstein \& Haber, 1965; Werner, 1935) abundantly demonstrates the existence of complex interactive events across the temporal dimension as well.

Up to now, however, virtually all related research has focused on the physical parameters involved in such interactions-luminance relations, adaptation levels, interstimulus intervals, proximity of borders, etc.

The present study reports the existence of interactive events in visual processing arising from the symbolic meaning of stimulus components. Specifically, findings are reported which suggest that the presence of an emotional element in an array of otherwise neutral items inhibits the detectability of the neutral elements in a brief single-glance exposure of the whole array. This effect is called here cognitive masking.

*Support of this research by Grant No. 07-2120 from the Rutgers University Research Council is gratefully acknowledged.

\section{METHOD}

Three versions of a basic stimulus configuration were constructed, consisting of a circular array of eight discrete stimulus items plus a critical central item (see Fig. 1). The three stimulus versions were identical except for the central element, which comprised a swastika (Fig. 1), a Star of David, or a schematic window (the swastika with its "arms" extrapolated).

The Ss were members of the Rutgers Hillel Foundation (a Jewish organization), a group for which the emotional symbols, whether the negative swastika or the positive Star of David, could be expected to have particular salience. Separate groups of volunteers were tested on 3 successive nights, each with one of the three versions of the stimulus-swastika ( $22 \mathrm{Ss})$, Star of David (19 Ss), or window (18 Ss). All groups received the same treatment, except for the stimulus version employed.

Following a standard 1-min dark-adaptation interval, each group was exposed to its preassigned stimulus version for a 200 -msec duration. The display mechanism was a Wollensak shutter, set at maximum illumination, attached to a $500-\mathrm{W}$ slide projector. The experimental room was completely dark during the exposure.

Immediately following the stimulus exposure, the room lights were turned on and the Ss commenced the first of two separate detection reports, a forced recall procedure followed by a recognition measure.

Recall instructions were read prior to the stimulus presentation. Each $\mathrm{S}$ received a recall protocol consisting of an
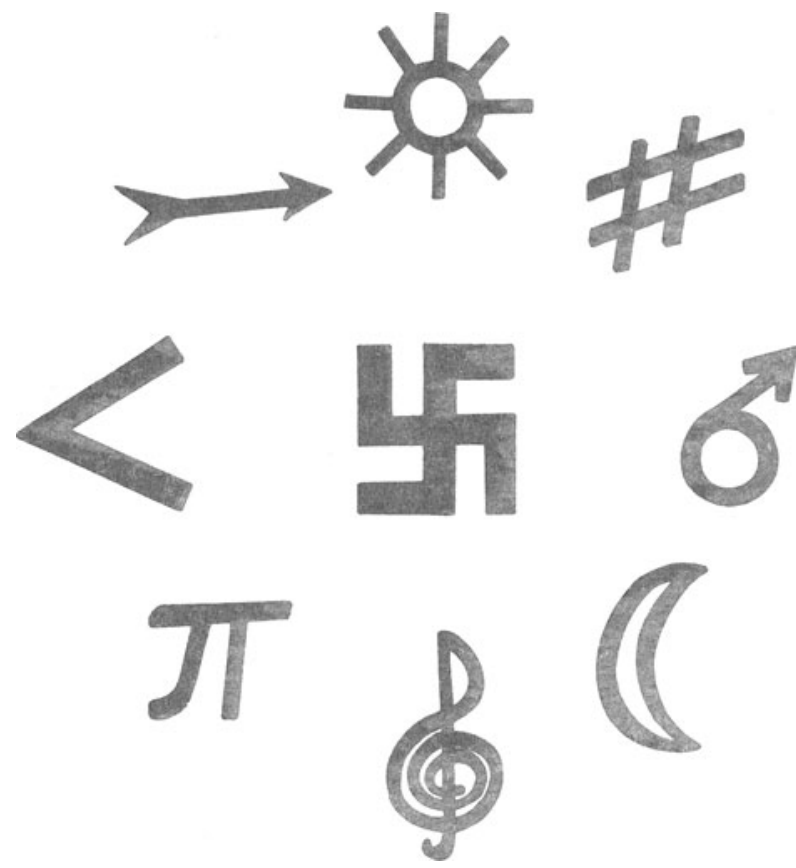

Fig. 1. One of the three basic stimulus versions employed: circular array of eight neutral items with swastika as the central item. All three versions of the stimulus were identical except for the central item, which was a swastika, a schematic window, or a Star of David. 


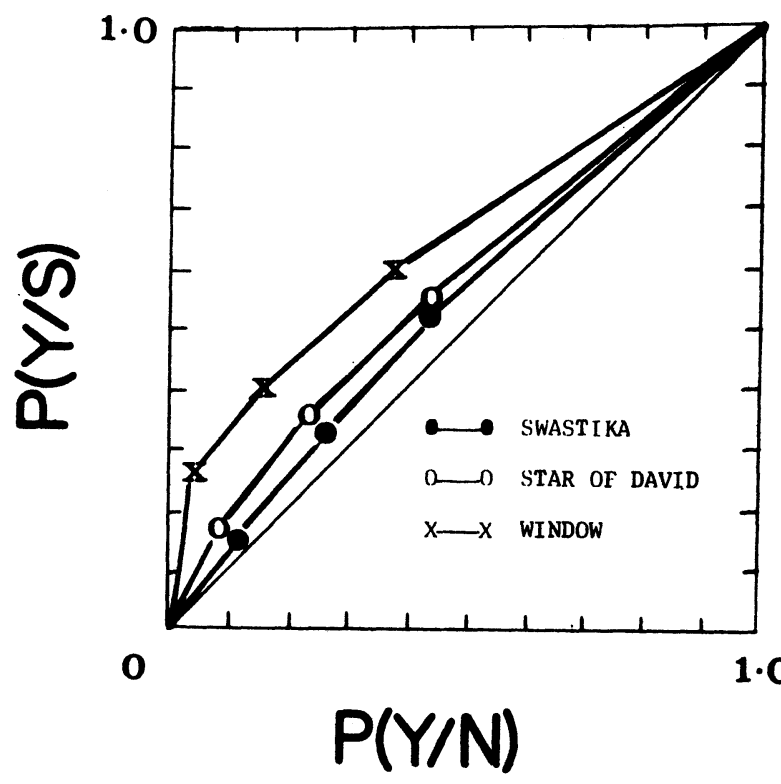

Fig. 2. Average ROC curves of the swastika, Star of David, and window groups for the identical eight neutral items.

array of nine empty circles arranged in the pattern of the stimulus elements to be displayed. The Ss were instructed that as soon as the lights were turned on following the stimulus flash, they were to fill in as many of the empty circles as they could with perceived elements of the stimulus. Once the stimulus had been presented and all Ss had completed their initial recall of the display, additional recall instructions were delivered: Ss were told to fill in each of the remaining blank (unfilled) circles with a nonrepeating guess of the unreported stimulus items. The recall scores for all Ss are, therefore, based on the same number of total responses, i.e., nine, comprising freely recalled items plus guesses. Such a forced recall procedure minimized the possibility of report criterion differences arising between groups. Recall scores reported below are based on a simple count of correct items included in the recall protocol, regardless of position or whether the item had been guessed or freely recalled.

An independent recognition task followed the recall procedure. Once the recall protocols had been collected, the Ss were presented with a succession of 18 slides, each of which contained a single pictured item. Nine of the items had actually appeared in the original stimulus array; the other nine were distractor items. The order of the items, except for the last two, was random; the last two constituted the critical item and a distractor. Each slide was presented for $20 \mathrm{sec}$, during which time the Ss were required to report on a prepared recognition protocol (1) whether the item had or had not been part of the original stimulus display (yes or no) and (2) the degree of confidence, small or great, attributed to the yes-no report. This recognition procedure thus yielded a four-point rating scale of stimulus recognition (confident yes, doubtful yes, doubtful no, and confident no). These recognition ratings provide the data base from which receiver operating characteristic (ROC) functions could be extracted through techniques that are by now well known (e.g., Green \& Swets, 1966). Such ROC functions are the basis for the direct estimates of sensitivity or detection reported below.

\section{RESULTS AND DISCUSSION}

The specific empirical question posed by this study was whether processing of the eight noncritical neutral ("orbit") items-identical for all three groups-would be affected by the symbolic import of the critical central item. Both detection indicators employed, the forced recall and recognition rating measures, produced parallel affirmative data.

Out of the set of eight neutral orbit items, the three groups (swastika, Star of David, and window groups) correctly recalled an average of $1.27,1.37$, and 2.12 items, respectively. ${ }^{1}$ An analysis of variance on these data revealed the obtained group means to be significantly different $[F(2,55)=4.01, p<.05]$. A contrast analysis on the means of the two emotional groups vs the mean for the neutral group was highly significant $[F(1,55)=8.28, p<.01]$.

The above recall scores are based on correct recall of orbit items, irrespective of recall or nonrecall of the critical central item. Although prior to stimulus presentation Ss had been instructed to fixate upon the center of the screen, the position of the critical item during the stimulus display, not all Ss recalled this item. The proportion of Ss correctly recalling their central item was $17 / 22$ for the swastika group, $17 / 19$ for the Star of David group, and 10/17 for the window group. A further analysis of data was performed on this subset of Ss. Average recall of orbit items for only those Ss reporting the central item was $1.00,1.30$, and 1.80 for the swastika, Star of David, and window groups, respectively $[F(2,41)=2.84, p=.07]$. The contrast of interest (emotional vs neutral) proved to be significant $[F(1,41)=4.60, p<.05]$. Thus, the data based on the forced recall indicator of detection, whether performance on the critical item is disregarded or taken into account, suggests that an emotional stimulus element tends to disrupt the processing of contiguous neutral items.

The recognition indicator yielded similar results. ROC curves for each $S$ were extracted from recognition ratings of the eight orbit items and the eight counterpart distractors. (The last two items in the 18-item inspections set, the critical item and its distractor, were not included in the computations, since the issue of interest was the detection of the eight orbit items, not the detection of the whole display.) Figure 2 depicts average ROC curves for the three groups. Visual inspection suggests that sensitivity for the neutral orbit items was lower when the critical central item was emotional (swastika or Star of David) than when the central item was neutral (window). A distribution-free measure of sensitivity, $\mathrm{P}(\mathrm{A})$, the proportion of total area underneath an ROC curve (Green \& Swets, 1966), was computed for each S. Average P(A) values for the swastika, Star of David, and window groups were, respectively, $53 \%, 57 \%$, and $66 \%$. An analysis of variance on $\mathrm{P}(\mathrm{A})$ scores proved significant $[\mathrm{F}(2,56)=5.49$, $\mathrm{p}<.01]$. The relevant contrast analysis (emotional vs neutral) was also significant $[F(1,56)=9.87, p<.01]$.

The recall and recognition data are thus thoroughly consistent: An emotional component of a complex 
stimulus, whether pleasant or unpleasant, can disrupt the processing of neutral components in a single-fixation exposure of the full display.

Although the present study was conducted with ostensibly emotional critical items, it is by no means clear whether the effect is limited to emotional stimuli. It is not clear, for example, whether the emotional groups' poorer performance on the neutral items was due to the disrupting effects of emotionality per se on processing, or to the monopolization of processing time (attention) by the critical item, resulting in diminished processing of the remaining components. Further work to resolve some of these issues is now in progress. The important point to have emerged from the present study, however, is that interactions in visual processing are not restricted to physical effects; a component of a stimulus may mask another component not only through its physical parameters but also through its symbolic properties.

\section{REFERENCES}

Cornsweet, T. N. Visual perception. New York: Academic Press, 1970.

Green, D. M., \& Swets, J. A. Signal detection theory and psychophysics. New York: Wiley, 1966.

Kahneman, D. Methods, findings, and theory in studies of visual masking. Psychological Bulletin, 1968, 69, 408-425.

Lindsay, P. H. \& Norman, D. A. Human information processing. New York: Academic Press, 1972.

Raab, D. Backward masking. Psychological Bulletin, 1963, 60, 118-129.

Ratliff, F. Mach bands: Quantitative studies on neural networks in the retina. San Francisco: Holden-Day, 1965.

von Békésy, G. Sensory inhibition. Princeton, N.J: Princeton University Press, 1967.

Weisstein, N., \& Haber, R. N. A U-shaped backward masking function in vision. Psychonomic Science, 1965, 12, 75-76.

Werner, H. Studies in contour. American Journal of Psychology, 1935, 47, 40-64.

\section{NOTE}

1. The recall score of one of the Ss belonging to the window group was excluded from the recall computations because of his failure, in spite of the instructions, to fill in all blank circles in his recall protocol.

(Received for publication October 8, 1972.) 